\title{
LAS CLÁUSULAS ANÓMALAS EN LA OBRA DE SILIO ITÁLICO: ESTUDIO MÉTRICO Y ESTILÍSTICO
}

This paper offers a study of the working of non-regular clauses in dactylic hexameter in Silius Italicus' Punica and makes a comparison between his procedures and the "model poets", mainly Virgil's.

Silius Italicus uses non-regular clauses much less frequently than Virgil, but with similar stylistic values. The most frequent clauses are those we will call $s i$ bona norint and armaque nostra, which usually appear related to enjambement. However, Silius Italicus's main innovation lies in his use of both types of clauses, combined with enjambement and chiasmus.

Teniendo en cuenta las características de los poetas postaugusteos que se vuelven definitivamente hacia Virgilio, Ovidio y Horacio como maestros, resulta siempre interesante su estudio con vistas a delimitar en qué aspectos sus poemas son innovadores o hasta qué punto dependen en su composición de la imitatio de los poetas modélicos. Estudios semejantes nos pueden orientar acerca de la evolución sufrida por la poesía desde la época clásica hasta la latinidad tardia. Desde esta perspectiva dirigimos nuestra consideración hacia la obra de Silio Itálico, del que sabemos que intentó deliberadamente la vuelta hacia la poesía de glorificación nacional' .

El poeta, erudito y estudioso de Virgilio, buscó su modelo en el gran maestro latino, aunque también se puede percibir la influencia de Ennio. Con todo, no pudo llegar a gran altura, pues su trabajo lo realiza$\mathrm{ba}$, al decir de Plinio el Joven, maiore cura quam ingenio.

Como objeto de nuestro estudio nos hemos propuesto la revisión, desde el punto de vista métrico-estilístico, de las cláusulas hexamétricas que podríamos denominar "anómalas" ${ }^{2}$, estableciendo la obligada comparación con el proceder del poeta modélico, Virgilio.

1 Con respecto a estos temas puede resultar instructivo el libro de $\mathrm{Cw}$. Mendell, Latin poetry. The Age of Rhetoric and Satira, Hamden 1967.

" Comúnmente se consideran cláusulas "normales" en el hexámetro dactílico las que responde a las disposiciones $3+2,2+3$ ó $2+1+2$. Aunque Nougaret en su céle- 
Resulta ya conocido que la cláusula del hexámetro en su aspecto métrico es la parte más cuidada del verso, donde el ritmo ha de reflejarse con mayor nitidez. En ese sentido, la tendencia innegable de los poetas hexamétricos latinos de época clásica es la de hacer uso, en este lugar del verso, de las disposiciones tipológicas que podríamos llamar «acostumbradas», reservando las "anómalas» como vehículo más apropiado para la expresión de un estilema, ya que su sola presencia provoca en el lector o en el oyente una llamada de atención ${ }^{3}$.

Por esta razón, estamos convencidos de que el análisis de las cláusulas «anómalas» puede desvelar las tendencias que guiaron al poeta en la constitución de su hexámetro, su deseo de originalidad y la fuerza de la imitatio en su técnica, si es que empleó este recurso.

Para proceder a la localización y al estudio de todas las cláusulas anómalas presentes en el autor, revisamos los 12.202 versos de que se componen los Punica. Las cláusulas de su hexámetro ofrecen habitualmente - como es lo esperado - las tipologías consideradas más regulares: $3+2,2+3,2+1+2$, con la consiguiente adecuación de acento de palabra y tiempo fuerte.

Comentaremos a continuación los versos que no se sujetan a esta regularidad, los que mejor reflejan, por tanto, las particularidades del autor. Señalaremos en cada caso los encabalgamientos que hubiere, dado que éste es fenómeno métrico-estilístico de primera importancia, como ha demostrado el profesor Holgado en su reciente obra sobre la frontera en el verso de Lucano ${ }^{4}$.

Comenzaremos nuestro estudio haciendo referencia a un tipo clausular casi desusado en Silio Itálico, ya que a lo largo de los más de 12.000 versos que se nos han transmitido, sólo lo emplea en dos ocasiones: se trata del final con monosílabo aislado.

Si la proporción de este tipo en la obra de Virgilio es claramente minoritaria $(0,36 \text { por } 100)^{5}$, podemos afirmar que en la de Silio presenta

bre Métrique admite como tales los tipos si bona norint, corpore qui se ot tribus et gens, en nuestra opinión estas distribuciones deben considerarse como realizaciones "anómalas", dado que su aparición presenta un índice de frecuencia muy bajo, y puede vincularse a rasgos estilísticos concretos.

${ }^{3}$ El tema ha sido estudiado detenidamente por nosotros en el trabajo presentado en febrero de 1988 como Tesis Doctoral, bajo el título Estudio métrico de la cláusula tipo armaque nostra en el hexámetro dactílico; dirigida por el profesor S. Mariner Bigorra, se publicó en Madrid 1988.

${ }_{4}^{4}$ A. Holgado, La frontera de verso en la Farsalia de Lucano: Estudio estilistico, Cádiz 1987.

$s$ Los datos estadísticos y, en general, los detalles que se refieren a la técnica de las cláusulas "anómalas» en los poetas de época clásica y preclásica - salvo cita en contrario - proceden del estudio presentado por nosotros como Tesis Doctoral y citado con anterioridad. 
visos de excepcionalidad, con un porcentaje que supone un 0,02 por 100 sobre el total de sus versos. Su única representación se descubre en:

$\begin{array}{lll}\text { XIII } & 862 & \text { ille deum gens } \\ \text { XVI } & 614 & \text { laudis agunt nos }\end{array}$

La ruptura de la norma en esta cláusula viene ofrecida por una doble anomalía, la tipológica y la acentual: la primera porque no respeta la presencia del bisílabo o trisílabo esperados en final de verso, y la segunda porque supone un broche de verso triplemente acentuado y «a destiempo", salvo en lo que respecta al tono que recae sobre el V longum.

Éstas son cláusulas que en Virgilio y Ovidio se revisten de una especial importancia desde el punto de vista estilístico, ya que suelen rodearse de figuras de dicción características, como el homeoteleuto, en exiguus mus (G. I 181), o ciertas cacofonías, así en oceano nox (En. II 250); con ello el término final queda claramente en relevancia.

Las secuencias en las que Silio Itálico utiliza esta distribución muestran claramente la impronta virgiliana.

En XIII 862 (ille deum gens) encontramos el recuerdo del conocido uigilasne deum gens $(E n . X 228)$ referido a Eneas. En el verso de Silio la expresión, con un sentido traslaticio, se aplica a César para poner de relieve su alto rango, estirpe de los mismos dioses.

Con respecto al verso XVI 614 (laudis agunt nos), creemos también encontrar una reminiscencia de Virgilio, por el recuerdo de los finales tempus agi res (En. V 638) y casus agat res (En. IX 723), en los que el mantuano sitúa una forma del verbo agere ante el monosílabo final.

Consideramos, por tanto, que este tipo de final en Silio Itálico es clara consecuencia de la imitatio uergiliana.

Una distribución muy poco aprovechada por Virgilio y poetas épicos posteriores - por lo que es lícito calificarla como cláusula anómala- es la que podemos representar por la secuencia $3+1+1$. En ella, el tan frecuente bisilabo final aparece sustituido por el conjunto de dos monosílabos que, en un buen número de casos, pueden presentarse formando «mot-métrique»: por ello, el tipo que consideramos puede casi identificarse con el habitual $3+2$. En alguna ocasión, sin embargo, el reconocimiento del susodicho "mot-métrique» resulta un tanto problemático, por lo que estos finales deben ser contemplados desde otra perspectiva.

En Silio Itálico encontramos, a lo largo de toda su obra, un total de 31 finales con la figura $3+1+1$, lo que representa un promedio del 0,25 
por 100 , inferior, por tanto, aunque próximo, al registrado por el autor modélico $(0,29$ por 100$)$.

Un gran número de estos finales se cierra con una forma del verbo esse; en ellos la vinculación entre los dos últimos vocablos es estrecha, y el acento recae necesariamente sobre el longum VI, con lo que la identificación entre $3+2$ y $3+1+1$, se hace plena.

En este caso estarian finales como:

$\begin{array}{lll}\text { II } & 576 & \text { aspera mors est } \\ \text { VI } & 518 & \text { perfide, nunc est? }\end{array}$

Con idéntica estructura hemos recogido: I 598, II 295, III 200, III 425, IV 780, VI 437, VII 35, VII 236, VIII 317, X 501, XVI 539.

En la misma línea estaría la serie de cláusulas que se cierran con una forma pronominal precedida, generalmente, de una preposición $o$ conjunción; entre ellas podemos señalar, a título de ejemplo:

$$
\begin{array}{lll}
\text { VI } & 588 & \text { parcite, si qua } \\
\text { XI } & 324 & \text { penatibus et me }
\end{array}
$$

Con características semejantes encontramos los versos siguientes: IX 35, X 70, X 286, X 314, XI 550, XIII 242, XV 362, XVI 88.

En estos casos, parece fácil el reconocimiento de la existencia de un solo "mot-métrique», y la conjetura de una acentuación acorde con la esperada: es decir, elevación del tono de voz sobre el VI longum.

Esta misma reflexión podríamos aplicarla a los versos:

$\begin{array}{lrl}\text { I } & 653 & \text { obruta iam nunc } \\ \text { IX } & 26 & \text { talia, qui te } \\ \text { XIII } & 655 & \text { hei mihi! nam cur } \\ \text { XVII } & 221 & \text { compos et hoc nunc. }\end{array}$

Atención diferente requieren, en cambio, los finales:

$\begin{array}{lll}\text { XI } & 529 & \text { altera iam lux // si talis ... } \\ \text { XII } & 261 & \text { obuia ni uis // Gestaris } \\ \text { XV } & 647 & \text { unica nunc spes, } \\ \text { XVII } & 379 & \text { praecordia. Sed lex // muneris }\end{array}$

En estos finales, aunque podríamos establecer la existencia de un solo mot-métrique, parece plausible suponer una discordancia acentual con respecto a la norma, ya que resulta más adecuado pensar que es el sustantivo el portador del acento. Por otra parte, los términos monosi- 
lábicos que cierran el verso parecen destinados a quedar en relevancia. Estaríamos, pues, muy cerca del final que denominamos "de monosílabo aislado", si bien se muestra algo más próximo a las estructuras de carácter regular, por cuanto que aquí no existe más que un desajuste acentual: un longum VI desprovisto de tono, mientras que en el tipo procumbit humi bos se mantiene además una breve acentuada en el quinto bíceps.

Los versos XII 261 y XVII 379 quedan también señalados por sendos encabalgamientos estrictos, en los que se quiebra el sirrema sustantivo-complemento determinativo; con la adición de este recurso, el monosílabo queda doblemente resaltado.

En la línea XI 529 podemos anotar un encabalgamiento de carácter laxo, por la disyunción de la oración principal y la subordinada condicional. Algo semejante percibimos en XV 647, por la disyunción del epíteto y del término al que va referido, Liuius, nombre propio que el poeta sitúa en el quinto pie del verso siguiente.

En cuanto a las cláusulas constituidas por un doble monosílabo final, casi asimilables a los tipos regulares, observamos que presentan también una cierta inclinación hacia el encabalgamiento, pues, de entre los 27 versos registrados, 18 ofrecen la disyunción de un sirrema, lo que supone un 66,6 por 100 sobre el total considerado. Podría argumentarse que este promedio resulta casi forzado por la presencia de conjunciones o elementos subordinantes en final de verso, pero es cierto que Silio Itálico en no pocas ocasiones busca el encabalgamiento por encima de esta condición, por ejemplo en los versos:

$\begin{array}{lll}\text { I } & 598 & \text { quo tua nunc sunt // fata loco? } \\ \text { VII } & 236 & \text { credite, non est // arduus }\end{array}$

No es, por tanto, el encabalgamiento una disposición «exigida» en este tipo de cláusula, sino elegida por el autor en la gran mayoría de los casos.

Otro final netamente señalado en Virgilio y poetas anteriores es el constituido por un término pentasilábico. En él se rompe la disposición tipológica habitual y, en cierta medida, la acentual, ya que, si bien se conserva claramente el acento sobre el VI longum, debe conjeturarse la existencia de un acento secundario, posible dada la longitud de la palabra, para mantener la regularidad.

Las cláusulas de este tipo en los poetas modélicos, Virgilio y Ovidio principalmente, recogen términos helénicos, nombres propios o vocablos consagrados por la poesía arcaica y, en consecuencia, especialmente señalados dentro del lenguaje poético. 
Silio Itálico, en estas cláusulas, parece haber seguido fielmente las disposiciones modélicas, pues los dos ejemplos que nos ofrece se acogen a la normativa heredada.

En I 203 encontramos aetheriasque, adjetivo de origen helénico, y en IV 160 quadrupedantum, término de carácter onomatopéyico que ya Ennio y Virgilio eligieron para ocupar este lugar del verso.

Podríamos, pues, asegurar que también con esta distribución Silio Itálico está practicando la imitatio uergiliana.

En cuanto a los finales de estructura tetrasilábica, podemos encontrar dos tipos con características muy diferentes: primero, el constituido por un término dispondaico, y segundo, el formulado con un vocablo cuya disposición cuantitativo-silábica coincide con la figura del jónico "a minore».

Las cláusulas integradas por un término dispondaico fueron ampliamente utilizadas por Catulo para acercar sus versos a las maneras de los neotéricos ${ }^{6}$, y, posteriormente, por Virgilio y Ovidio con intención semejante. Silio Itálico es autor remiso en su empleo, pues no encontramos en toda su obra más que tres ejemplos $(0,02$ por 100$)$, frente al 0,12 por 100 de Virgilio y al 0,20 por 100 de las Metamorfosis.

Los versos que presentan tal disposición clausular son los siguientes:

$\begin{array}{lll}\text { II } & 314 & \text { Apenninus } \\ \text { IV } & 742 & \text { Apenninus } \\ \text { VIII } & 621 & \text { Hellespontus }\end{array}$

Ambos términos aparecen ocupando la cláusula del hexámetro por primera vez en la obra de Ovidio, concretamente en Met. II 226 (Apenninus) y en Met. XIII 407 (Hellespontus), con lo que la imitatio debe anotarse esta vez en el débito del poeta de Sulmona.

Con respecto a los finales en forma de jónico «a minore», debemos hacer una distinción: cabe señalar en primer lugar aquellos que realizan una perfecta homodinia, bien por ir precedidos de un monosílabo, bien porque, precedidos de un polisílabo, provocan una elisión que hace coincidir el acento de palabra con el $\mathbf{V}$ longum: son las distribuciones que podríamos denominar, genéricamente, di genuerunt e inceptosque hymenaeos.

En línea opuesta estarian aquellas cláusulas constituidas por un vocablo con forma de jónico «a minore» precedido de otro polisílabo ca-

${ }^{6}$ Es un dato innegable la frecuencia de estë tipo de finales - con una proporción del 4,43 por 100 - en los poemas no epigramáticos, los Carmina docta, en los que el poeta intenta la imitatio de la métrica helenística. 
paz de provocar la heterodinia sobre el quinto pie: seria la distribución denominada gemitu lacrimisque.

Silio Itálico es asimismo parco al adoptar estas disposiciones, pues, a lo largo de toda su obra, encontramos sólo cuatro ejemplos, tres homodínicos y uno heterodínico, con una proporción del 0,03 por 100 , frente al 0,37 por 100 de Virgilio.

También en este caso parece el mantuano inspirador de nuestro poeta, ya que Ovidio construye regularmente este tipo de cláusulas con finales heterodínicos, seguramente porque deseaba entroncar con las técnicas helénicas, y la homodinia podía considerarse como un elemento rítmico complementario dentro de la poesía latina, pero absolutamente inoperante en la griega. Podemos añadir, además, que la terminología utilizada por Silio Itálico en este tipo de cláusulas recuerda claramente otras de la obra virgiliana.

En este sentido, superumque hominumque (Pun. I 152) podria ser reminiscencia de hominumque deumque (En. I 229), evocación a su vez del áv$\delta \rho \tilde{\omega} v \tau \varepsilon \theta \varepsilon \tilde{\omega} v \tau \varepsilon$ homérico.

Y los finales primoque Hymenaeo (Pun. III 64), lateque Hymenaeo (Pun. V 22) y Tyrios Hymenaeos (Pun. XVII 79) traen a nuestra memoria otros del mantuano que se cierran con el mismo vocablo, hymenaeus. Éste es término presente ya en Catulo, donde se repite trece veces, nueve de ellas formando parte del estribillo del Carmen 62 bajo la forma o hymenaee.

En consecuencia, pensamos que Silio Itálico continúa utilizando la técnica helenizante de sus predecesores, pues su fraseología sugiere la evocación de la Hélade, pero desatiende el enfoque característico de Ovidio hacia la heterodinia, quizá porque en la época de Silio eran mejor acogidos los finales que presentaban coincidencia de acento de palabra y tiempo fuerte.

Consideraremos a continuación el uso que hace el autor de los Puni$c a$ de una de las cláusulas anómalas más frecuentes en Virgilio: se trata de la que genéricamente podríamos representar con el lema si bona norint, o la secuencia numérica $1+2+2$.

La distribución que examinamos ahora parece que combina una doble anomalía, la tipológica y la acentual: la primera, porque prescinde del obligado trisílabo y lo realiza de forma más entrecortada; la segun$\mathrm{da}$, porque ofrece dos acentos contiguos sobre el quinto pie, aminorando así o sustituyendo la relevancia del acento sobre el V longum.

Con todo, ambas alteraciones pueden o reducirse al máximo, en grupos con la configuración tu quoque uotis, o aminorarse al menos, co- 
locando sobre el quinto bíceps un término gramatical de débil tonicidad.

Esta cláusula tripartita, según los estudios de Soubiran ${ }^{7}$, muestra en el mantuano estrecha vinculación con la diéresis bucólica y el encabalgamiento, pudiendo considerarse esta doble relación como rasgo original y creación virgiliana, ya que constituye un fenómeno no detectado en ninguno de los poetas anteriores.

Para exponer más fácilmente las características de las cláusulas tipo si bona norint en Silio Itálico, iremos señalando, por una parte, aquellos rasgos que parecen comunes con los de la obra virgiliana, y por otra, aquellos que parecen más específicos de nuestro autor. Anotaremos en cada caso la localización de los versos y el texto de la cláusula $y$, cuando corresponda, ofreceremos un breve comentario sobre su valoración y cualidades desde el punto de vista de la estilística.

Como en el poeta modélico, la gran mayoría de estas cláusulas comienzan un nuevo miembro de frase, realizándose así con nitidez la puntuación bucólica, recurso que, como es sabido ${ }^{8}$, sirve habitualmente para destacar el grupo métrico final del verso. Las únicas excepciones a esta casi norma las tenemos en los versos:

$\begin{array}{lll}\text { II } & 332 & \text { non latet hostis. } \\ \text { IV } & 368 & \text { at non dabat ultra } \\ \text { V } & 154 & \text { meus, heu! meus atris } \\ \text { IX } & 529 & \text { non placet Irim? } \\ \text { XI } & 356 & \text { namque haec tibi ferrum, }\end{array}$

En los versos IV 368, V 154 y XI 356 el nuevo kolon comienza con la cesura heptemímera, mientras que en II 332 se remonta hasta la pentemímera, y en IX 529 no se percibe solución de continuidad a lo largo de toda la línea. En resumen, podemos considerar que, en este tipo, el número de cláusulas vinculadas con la diéresis bucólica alcanza un porcentaje del 92,17 por 100 sobre el total considerado.

De modo semejante a la práctica registrada en el autor de la Eneida, la cláusula que consideramos se presenta unida al encabalgamiento; las únicas excepciones las encontramos en los versos:

$\begin{array}{lll}\text { II } & 332 & \text { non latet hostis. } \\ \text { III } & 586 & \text { hinc tibi Paulus, // ... } \\ \text { VII } & 114 & \text { at tamen audet. } \\ \text { IX } & 335 & \text { hi sude pugnas, / ... }\end{array}$

7 J. Soubiran, "Intremere omnem et si bona norint: recherches sur l'accent de mot dans la clausule de l'hexamètre latin», Pallas 8, 1959, pp. 23-56.

${ }^{8}$ J. Marouzeau, Traité de stylistique latine, París 1962, p. 306. 


$\begin{array}{lll}\text { IX } & 529 & \text { non placet Irim? } \\ \text { XI } & 169 & \text { huc, age, adeste. } \\ \text { XV } & 446 & \text { dumque ea fatur, } \\ \text { XVI } & 393 & \text { haec ubi dicta, }\end{array}$

En los vv. II 332, VII 114, IX 529 y IX 169 la cláusula cierra un período con pausa fuerte, mientras que las de III 586 y IX 335 forman parte de sendas enumeraciones (señaladas gráficamente con el signo // ...). En el caso de los vv. XV 446 y XVI 393 la cláusula se ocupa con una oración subordinada temporal que se vincula por el sentido y la sintaxis al verso siguiente: en ese sentido podriamos hablar de encabalgamiento laxo.

Como se percibe claramente en Virgilio, también en Silio Itálico la relación entre el tipo clausular $1+2+2$ y el encabalgamiento se hace casi habitual, pues el número de versos que presentan esta distribución, pero no rompen la frontera versal, supone solamente un 12,69 por 100 sobre el conjunto de los que se examinan.

Dejando aparte los 8 versos considerados anteriormente, de los 63 que responden a esta ordenación, 35 se proyectan con un encabalgamiento simple - lo habitual en el poeta modélico- y 20 con un encabalgamiento complejo.

Dentro de los encabalgamientos simples, la disyunción más frecuente se realiza por la ruptura del sirrema integrado por el sustantivo y su determinante, ya se trate de un adjetivo de cualquier género o de un complemento determinativo nominal.

Éste sería el caso de los versos siguientes:

\begin{tabular}{|c|c|c|}
\hline I & 60 & his super, aeui // flore uirens \\
\hline I & 342 & en bona uirtus // primitiaeque ducis \\
\hline$* 1$ & 536 & et super ipsas $/ / \ldots$ pugnas \\
\hline IV & 96 & ac simul acer $/ / \ldots$ hinnitus \\
\hline IV & 565 & haud secus acer // ... Allius \\
\hline V & 312 & nunc ferus alta $/ / \ldots$ ceruice \\
\hline VI & 645 & atque ubi latis $/ / \ldots$ in campis \\
\hline VII & 82 & hoc tibi, diua, // ... uelamen \\
\hline$*$ VII & 205 & hic ubi primo // ... Phoebo, \\
\hline IX & 128 & haud tua, nate, // fraus \\
\hline IX & 306 & aut sator aeui // ... Iuppiter (v. 308) \\
\hline *IX & 600 & et facis atrae, $/ / \ldots$ sulphure \\
\hline $\mathrm{X}$ & 421 & is mala bello // pectora \\
\hline XI & 93 & an tua, Virri, //... sors, \\
\hline XI & 177 & ille ego sanguis // Dardanius \\
\hline$* \mathbf{X I}$ & 320 & hic erit ille, $/ / \ldots$ ensis \\
\hline XIII & 280 & et bona uirtus $/ / \ldots$ punita \\
\hline
\end{tabular}




$\begin{array}{ccl}\text { XIV } & 550 & \text { mox sua ponto } / / \ldots \text { unda } \\ \text { * XIV } & 620 & \text { ut grauis ergo // .. Sirius } \\ \text { XV } & 713 & \text { ut caua currunt } / / \text { nubila, } \\ \text { XV } & 732 & \text { hei mihi quanta } / / \ldots \text { dextra, }\end{array}$

En la mayoría de los casos encontramos un encabalgamiento suave y desinente: a veces se prolonga de tal manera su figura, que la segunda parte del sirrema no llega a exponerse hasta la cláusula del verso encabalgado, con lo que se realiza una "correspondencia vertical»" que sirve para poner en mayor relevancia los términos unidos por el sentido, correspondencia que se subraya, en ocasiones, con la figura del homeoteleuto. Este fenómeno ha sido señalado en la relación de cláusulas que hemos presentado con la adición de un asterisco.

Conviene señalar que la cláusula del verso IX 306, con encabalgamiento abrupto incipiente, queda en tensión hasta la línea 308 en que se introduce el nombre del creador, Júpiter - que se estaba esperando-, tras saltar sobre una oración subordinada que se desarrolla a lo largo del verso 307.

El fenómeno del braquistiquio, aunque no es muy frecuente, aparece claramente en el verso XV 713. Ésta es una figura que el poeta utiliza para enfatizar un vocablo determinado o subrayar el tono de un pasaje. En este caso se trata de esta última opción: el autor narra el arrojo de la juventud romana en la lucha y, para ello, utiliza el recurso de la comparación, con una expresión que va creciendo gradualmente: primero es simplemente un sustantivo, ut torrens, ut tempestas, después el sustantivo con su determinante, ut flamma corusci fulminis, en tercer lugar una oración simple ut Borean pontus fugit, y por fin, con el mayor énfasis posible, el conjunto de una oración principal y su subordinada: ut caua currunt // nubila, cum pelago caelum permiscuit Eurus.

Otro tipo de encabalgamiento consiste en la disolución de un sirrema integrado por el verbo más su sujeto, complemento o determinante, como en los versos siguientes:

$\begin{array}{lll}\text { II } & 171 & \text { at uelut ales // ... rapitur } \\ \text { III } & 573 & \text { hac ego Martis // ... paro } \\ \text { IV } & 368 & \text { at non dabat ultra // Clotho } \\ \text { VII } & 200 & \text { iam pede risum // ... moues } \\ \text { XII } & 291 & \text { qui dare terga // ... potes? } \\ \text { XIII } & 188 & \text { et super esse // ... quemque ducem } \\ \text { XV } & 89 & \text { huc, age, paulum // aspice }\end{array}$

9 Cf. a este respecto el artículo de A. W. de Groot «Le mot phonetique et les formes littéraires du latin", $R E L, 1934$, p. 124. 


$\begin{array}{lll}\text { XV } & 649 & \text { ite, agite, oro // sternite ductorem, } \\ \text { XVI } & 51 & \text { seu foret hostis // comminus, } \\ \text { XVII } & 187 & \text { o nihil umquam // crescere } \\ \text { XVII } & 549 & \text { aut latet idem // cur monstro? }\end{array}$

La mayor parte de estas cláusulas se prolongan en un encabalgamiento suave y desinente; tenemos, en cambio, braquistiquio en los versos XV 89, XVI 51 y XVII 549.

Aunque la norma en latín aboga por el enunciado del verbo en el final del periodo, en alguna ocasión es la forma verbal la que aparece en primer lugar: es alteración que el poeta propone, sin duda, en aras de una mayor expresividad.

Menos frecuentes son los encabalgamientos denominados oracionales, es decir, aquellos en los que queda pendiente una partícula subordinante; entre estos casos estarían:

$\begin{array}{lll}\text { IV } & 239 & \text { at Batus, amens // qui } \\ \text { XII } & 175 & \text { ast ubi iusso, // ... fundite } \\ \text { XIII } & 655 & \text { hei mihi! nam cur // ... fuere }\end{array}$

El caso más peculiar puede parecer el del verso IV 239, donde queda en suspenso un predicativo amens precedente del relativo qui, a cuya oración en realidad pertenece: queda así en relevancia la condición del soldado Batus, al que califica.

Singulares son también las estructuras de las cláusulas que cierran los versos:

$\begin{array}{lll}\text { IX } & 291 & \text { hinc Venus amens // hinc Vesta } \\ \text { XVII } & 284 & \text { hoc satis irae // interea, genitor }\end{array}$

En el v. IX 291 encontramos aquel tipo de encabalgamiento que Büchner ${ }^{10}$ denomina en bikolon, ya que se evita la separación de dos kola contiguos, cuya unidad se señala por la anáfora y el paralelismo.

En el v. XVII 284 la cláusula contiene las palabras iniciales del parlamento de Venus a su padre: su primera frase quiebra la pausa al introducir un adverbio y a continuación el vocativo que designa al destinatario de la invocación.

Más interesantes resultan, desde nuestro punto de vista, aquellas cláusulas en que Silio Itálico proyecta un encabalgamiento complejo

${ }^{10} \mathrm{~K}$. Büchner, Beobachtungen über Vers- und Gedankengang bei Lukrez, Berlín 1936, p. 47 ss. 
o geminado ", y que muy frecuentemente adorna con la figura del quiasmo, tan querida por el autor, como ha señalado recientemente G. Flammini ${ }^{12}$. Ésta es disposición que no se ha detectado en autores de época clásica y que bien podría, por tanto, ser creación del autor de los Punica.

Los versos que presentan encabalgamiento geminado son los siguientes:

$\begin{array}{lrl}\text { * I } & 48 & \text { ac sua largo // stagna ... tabo } \\ \text { I } & 598 & \text { quo tua nunc sunt // fata loco? } \\ \text { III } & 605 & \text { hic fera gentis // bella Palaestinae } \\ \text { V } & 154 & \text { meus, heu! meus atris // ... frater ripis } \\ \text { V } & 155 & \text { at meus alta // ... stagna ... natus } \\ \text { XVI } & 47 & \text { hic fera gentis // more ... proelia } \\ \text { XVI } & 664 & \text { hoc sator aeui // Iuppiter aeterni }\end{array}$

En estos casos, los sirremas que se escinden están compuestos por un sustantivo y su determinante, generalmente una forma adjetival, aunque en el verso XVI 47 debemos admitir la equivalencia entre el adjetivo y el complemento determinativo fera / gentis. La situación en que aparecen los elementos de la cláusula con respecto a los correspondientes en el verso encabalgado, se plasma en dos posiciones quiasmáticas diferentes (de ordenación entrecruzada y de ordenación concéntrica) ${ }^{13}$, que iremos señalando en cada caso; en ocasiones el autor recurre además al ornato de la correlación vertical, cuando los términos del sirrema que ocupan el verso encabalgado aparecen - al menos uno de ellos - en la cláusula. Es dato que hemos señalado con el correspondiente asterisco en la lista de las cláusulas que hemos ofrecido.

Presentan estructura quiasmática de ordenación entrecruzada los versos I 48, III 605, V 154 y XVI 664 y, por el contrario, estructura quiasmática de ordenación concéntrica los versos I 598, V 155 y XVI 47.

Presentan un encabalgamiento complejo no geminado las cláusulas de las líneas:

"Con respecto a la distinción entre "encabalgamiento complejo» y "encabalgamiento geminado", nos acogemos a la propuesta del profesor Holgado en $o$. $c$., p. 43.

${ }_{12}$ Cf. a este respecto el artículo de G. Flammini, «Il chiasmo in Silio Italicoi, GIF N.S. 14, 1983, pp. 85-102.

${ }_{13}$ Silio Itálico, al menos en vinculación con estas cláusulas denominadas "anómalas» no presenta la posición quiasmática considerada normal (AB/BA), sino que se decanta hacia las figuras más elaboradas que, en el esquema más sencillo, podríamos simbolizar como AA/BB (de ordenación entrecruzada) y AA/BB (de ordenación concéntrica). 


$\begin{array}{lll}\text { I } & 400 & \text { et ferit alte // insurgens ... aera } \\ \text { III } & 619 & \text { huic sua Musae // sacra ferent } \\ \text { VIII } & 169 & \text { nec, quae tibi fraudes // tendantur ... cernis? } \\ \text { VIII } & 214 & \text { eia, age, segnes // rumpe moras } \\ \text { * VIII } & 301 & \text { aut ego multo // ... didici ... Marte. } \\ \text { IX } & 151 & \text { at miser, imo // pectore suspirans } \\ \text { XI } & 318 & \text { hoc ego bellum // conficere ... paro } \\ \text { * XI } & 356 & \text { namque haec tibi ferrum, // ... per uiscera ferrum } \\ \text { XII } & 513 & \text { hoc iter Alpes // hoc ... strauere } \\ \text { XIII } & 818 & \text { haec quoque castae } / / \ldots \text { ualuit mentis } \\ \text { XV } & 444 & \text { en age, miles, // .. talis rue (446) }\end{array}$

También en este tipo de disyunciones se puede percibir la disposición en quiasmo de ordenación entrecruzada y concéntrica. A las primeras pertenecerían VIII 214, VIII 301, XI 356, XII 513 y XIII 818; entre las segundas reseñamos: I 400, III 619, VIII 169, IX 151, XI 318 y XV 444.

Los sirremas que se diluyen en estas cláusulas no presentan suficiente uniformidad como para intentar la sistematización.

Merece quizá la pena subrayar la presencia de los encabalgamientos encadenados que se formulan en los versos XI 356-358: con la cláusula de la línea 356 se inicia una frase que no terminará hasta la pentemimera de la 358; un solo sintagma - per haec uiscera nostra- se reparte en tres versos como para estrechar su relación, interés que se recoge también con la presencia de la anáfora ferrum / ferrum y con la correspondencia vertical que se establece entre los términos que ocupan los pies $\mathrm{V}$ y VI de los dos versos consecutivos 356 y 357: haec / uiscera y ferrum / ferrum.

En el verso XV 444 el enunciado de la cláusula, que forma parte de la alocución a los soldados y comienza con una interjección de ánimo, queda en suspenso con un encabalgamiento abrupto incipiente; su sentido no se completa hasta el verso 446 - con las palabras talis rue-, pasando por encima del verso 445: éste recoge dos complementos de dirección y la primera parte de la comparación introducida por qualis.

Tras la revisión de las 63 cláusulas que en Silio Itálico responden a la distribución si bona norint, hemos podido comprobar que, aunque su frecuencia es notablemente inferior a la virgiliana $(0,51$ por 100 en Silio, frente al 1,2 por 100 del mantuano), su técnica corre pareja a la del autor de la Eneida.

Efectivamente, el tipo $1+2+2$ en un 92,06 por 100 de los casos aparece vinculado a la diéresis bucólica, y en un 87,3 por 100 deja percibir el desajuste entre la pausa versal y la de sentido. Merece aquí la pena subrayar que en 18 ejemplos $(26,47$ por 100$)$ el encabalgamiento se hace 
doble y se adorna con la figura del quiasmo, tan grata y tan utilizada, por lo general, en Silio Itálico.

En ocasiones, los términos de la disyunción se sitúan en la cláusula de los dos versos consecutivos, lográndose así una nueva ornamentación, por la "relación vertical» que se establece entre ellos, y una mayor relevancia de los vocablos en juego:

Es figura que encontramos, a título de ejemplo, en:

$\begin{array}{lll}\text { I } & 48 & \ldots \text { ac sua largo // } \\ \text { I } & 49 & \text { stagna ... turbida tabo // }\end{array}$

En la mayoría de los casos la disyunción se establece entre los miembros de un sirrema compuesto por adjetivo y sustantivo; en algún momento la disyunción se hace menos sensible, más laxa, ya que el vínculo se establece entre los dos miembros de una enumeración o entre una oración principal y su subordinada. A veces la tensión se diluye por la inserción de un nuevo verso - que distrae la atención del lector o del oyente - entre el enunciado de los dos términos de un encabalgamiento.

Parece, pues, evidente, a tenor de las cifras obtenidas en nuestro recuento, que la tipología $1+2+2$ era utilizada por Silio Itálico para configurar un final hexamétrico caracterizado por la puntuación bucólica (92,06 por 100 de los casos) y el encabalgamiento $(87,30$ por 100 de los casos). Encontramos en esta disposición, que se hace ya típica en nuestro poeta, un rasgo más de la imitatio uergiliana, pues, como ya advertimos, ésta parece ser una fórmula acuñada por el autor de la Eneida.

La última de las cláusulas tradicionalmente consideradas anómalas que hemos podido registrar en los Punica, es la que responde al lema genérico armaque nostra. Es distribución frecuente en Virgilio, que la combina en más del 80 por 100 de los casos con la figura métrico-estilística del encabalgamiento.

La anomalia de este tipo clausular responde a la irregular distribución de acentos que sustenta, pues la presencia de la enclítica hace bascular el tono sobre la primera breve del bíceps $\mathbf{V}$, en lugar de situarlo sobre el longum correspondiente; en ocasiones el acento se desplaza aún más, sobre la segunda breve del V bíceps: es el caso de la figura representada con el lema ardentiaque ossa, minoritaria pero presente también en Virgilio; tal distribución se recoge cuando el término con enclítica elide su última vocal sobre la inicial del vocablo siguiente.

A continuación proponemos el texto y la localización de las cláusulas que en los Punica responden a tal configuración, con un breve co- 
mentario que revela las características de estos finales, sobre todo desde el punto de vista de la estilística.

De los 89 versos que presentan esta distribución clausular en Silio Itálico un número muy elevado (66, que supone un 74,15 por 100 sobre el total) se continúa en encabalgamiento, según los usos del modelo.

Las cláusulas que respetan la frontera versal, o bien cierran un período con pausa fuerte o bien forman parte de una enumeración. Esta última disposición la señalaremos, como ya indicamos anteriormente, con el signo $/ / \ldots$.

Son las siguientes:

\begin{tabular}{|c|c|c|}
\hline I & 117 & Tarpeiaque saxa. \\
\hline I & 170 & tormentaque portant \\
\hline I & 481 & seruataque iura, \\
\hline I & 613 & neglectaque mensa // ... \\
\hline I & 629 & Alpinaque gaesa. \\
\hline II & 295 & disiectaque pax est. \\
\hline II & 298 & Massylaque uates. \\
\hline II & 650 & laudandaque monstra // ... \\
\hline III & 256 & Sarranaque Leptis // ... \\
\hline III & 367 & Aetolaque Tyde. \\
\hline III & 390 & raptaque pascunt. \\
\hline IV & 50 & armaque iungit, \\
\hline IV & 774 & lacerataque crines, \\
\hline V & 82 & Tarpeiaque saxa, // ... \\
\hline VI & 364 & horaque cursus, $/ / \ldots$ \\
\hline VI & 468 & Sarranaque Iuno // ... \\
\hline VII & 513 & optandaque Poeno, \\
\hline XI & 345 & Thrasymennaque busta // ... \\
\hline XII & 193 & horaque nostra est. \\
\hline XIV & 567 & pennataque Siren. \\
\hline XIV & 579 & Cadmeaque Sidon. \\
\hline XV & 264 & digestaque praeda: \\
\hline XVI & 105 & hebetataque tela. \\
\hline
\end{tabular}

Los vocablos que unidos a la enclítica aparecen en la cláusula formando parte de una enumeración, suelen constituir el segundo elemento de una secuencia que comienza en el principio de la línea y continúa después, incluso, a lo largo de uno o dos versos.

Como muestra de estos tipos, y especialmente destacables desde el punto de vista estilístico, ofrecemos el conjunto de los versos III 256258.

Sabratha tum Tyrium uulgus Sarranaque Leptis

Oeaque Trinacrios Afris permixta colonos

et Tingim rapido mittebat ab aequore Lixus. 
En el pasaje, los sujetos van ocupando los lugares destacados en el verso, el comienzo y el final, mientras que la forma verbal se asienta en la última línea tras la pentemimera, recogiendo la expresión, pero dejando el énfasis para los nombres que designan a los aliados de Aníbal.

De las restantes cláusulas que presentan la estructura armaque nostra - como ocurría con el tipo si bona norint-, la mayor parte $(54=$ 60,67 por 100 ) se construye con encabalgamiento sencillo, y un número menor $(12=13,48$ por 100$)$ pero aún significativo, con un encabalgamiento complejo o geminado.

En los encabalgamientos sencillos el sirrema más usual es el formado por el sustantivo y su determinante. Anotamos a continuación los 27 ejemplos recogidos con esta distribución:

$\begin{array}{llll}\text { I } & 102 & \text { aspersaque tabo // limina } & \text { B } \\ \text { I } & 169 & \text { turbataque luctu // .. gens } & \text { A } \\ \text { I } & 253 & \text { permixtaque nimbis // fulmina } & \text { B } \\ \text { I } & 592 & \text { fractaque anhelant // aequora } & \text { B } \\ \text { II } & 73 & \text { Pangaeaque lustrant // ... nemora } & \text { S.D. } \\ \text { II } & 331 & \text { armaque tantum // .. sumpta } & \text { A } \\ \text { II } & 447 & \text { condensaque cingunt // agmina } & \text { A } \\ \text { III } & 47 & \text { nullaque circa // litora } & \text { B } \\ \text { III } & 90 & \text { suppostaque caelo // saxa } & \text { A } \\ \text { III } & 272 & \text { scelerataque sucis // spicula } & \text { A } \\ \text { III } & 666 & \text { nemorosaque regna // cornigeri Iouis } & \text { A } \\ \text { IV } & 656 & \text { percussaque longe // .. saxa } & \text { A } \\ \text { V } & 203 & \text { disiectaque crinem // ... Venus } & \text { A } \\ \text { VI } & 213 & \text { scutataque raptim // ... manus } & \text { S.D. } \\ \text { VIII } & 397 & \text { detritaque bellis / Suessa } & \text { B } \\ \text { VIII } & 470 & \text { illusaque tauro // Pasiphaë, } & \text { B } \\ \text { IX } & 399 & \text { atraque manant // .. lumina } & \text { S.D. } \\ \text { X } & 447 & \text { dictataque iurant // sacramenta } & \text { A } \\ \text { XII } & 1 & \text { fecundaque nimbis // tempora } & \text { B } \\ \text { XII } & 373 & \text { pallidaque intus // arua } & \text { A } \\ \text { XII } & 380 & \text { aduersaque late // agmina } & \text { S.D. } \\ \text { XIII } & 72 & \text { Simoentaque nobis // ... sudatum } & \text { A } \\ \text { XIII } & 819 & \text { uenturaque dixit // regna } & \text { A } \\ \text { XIV } & 611 & \text { inhumataque late // corpora, } & \text { B } \\ \text { XV } & 377 & \text { laxataque luctu / .. arma } & \text { S.D. } \\ \text { * XV } & 767 & \text { demersaque in undam // ... cadauera } & \text { S.D. } \\ \text { XVII } & 110 & \text { accitaque regno // ... pubes } & \text { S.D. }\end{array}$

De estas 27 cláusulas, ocho se construyen con la figura que denominamos braquistiquio ${ }^{14}$, recurso que, en este caso, conviene distinguir del

14 Adoptamos en este caso la terminología de A. Quilis en Métrica española, Madrid 1983, p. 76, adaptándola a nuestras condiciones dentro de la lengua latina y estableciendo una leve diferencia con respecto a la propuesta de A. Holgado en $\mathrm{La}$ 
llamado encabalgamiento abrupto desinente. El braquistiquio se origina cuando el sirrema en disyunción termina con el primer dáctilo, generando una pausa en la elocución y el comienzo de un nuevo miembro de frase. En la lista de versos que hemos presentado, esta disposición aparece señalada con una «B».

En doce cláusulas señalamos la presencia de un encabalgamiento abrupto desinente, es decir aquel en el que el sirrema encabalgado completa su sentido ante la cesura pentemímera o trocaica. Señalamos tal ordenación con la adición de una "A» sobre el margen derecho.

En las siete restantes, el encabalgamiento iniciado en los últimos pies del verso se continúa en el siguiente, más allá de la cesura pentemímera. Esta ruptura de la pausa versal, de carácter suave y desinente, aparece señalada en nuestro texto con las letras «S.D.»

Conviene destacar que Silio Itálico hace uso del braquistiquio en esta fórmula para resaltar pasajes de carácter patético o sobrenatural: así escuchamos el fragor de las olas que se rompen (I 592); contemplamos las puertas salpicadas por la sangre corrompida (I 103), la ciudad de Suesa triturada por la guerra (VIII 397), o los cadáveres insepultos que yacen por doquier (XVI 611).

Por otra parte, se nos hace presente Júpiter cuando lanza sus rayos (I 253); Aníbal, que ve cosas maravillosas (III 47); Pasífae, subyugada por el Toro (VIII 470); o Invierno cuando esconde sus sienes tras las nubes (XII 1).

Es evidente que el autor quiere jalonar estos pasajes especialmente significativos con la presencia del braquistiquio y de una cláusula anómala - precisamente la que ofrece un desequilibrio acentual - para que aparezcan totalmente resaltados.

Conviene además subrayar la presencia de una relación vertical entre los dos términos del sirrema en disyunción en los versos XV 767 y 768 , donde los vocablos demersa y cadauera ocupan el lugar tras la cesura heptemímera.

Señalaremos a continuación aquellos versos en los que los términos en disyunción están constituidos por una forma verbal y su sujeto o complemento directo; en algún caso, sin embargo, cabe anotar la presencia de un predicativo, un complemento agente, o un circunstancial estrechamente vinculados con el verbo en cuestión.

frontera... El profesor Holgado parece identificar braquistiquio y encabalgamiento abrupto desinente, mientras que para nosotros el braquistiquio representa la estructura más breve dentro del abrupto, de modo que no sobrepasa los cinco tiempos y presenta un carácter más tajante o incisivo. 


\begin{tabular}{|c|c|c|c|}
\hline I & 9 & conuentaque patrum $/ /$... fregere & S.D. \\
\hline I & 70 & Saturniaque arua // addiderat & B \\
\hline I & 618 & armaque rapta $/ / \ldots$ ducibus & $\mathbf{A}$ \\
\hline II & 33 & praeruptaque saxa // scandatis & A \\
\hline II & 642 & deceptaque uisis // ... clamat, & S.D. \\
\hline III & 705 & extremaque fati // deposcis & B \\
\hline IV & 43 & consultaque belli // stare probat & A \\
\hline IV & 579 & resolutaque ripa // implicat & B \\
\hline *V & 628 & tremebundaque tela, // ... torquebat & S.D. \\
\hline VII & 379 & Romanaque tela // euasisse & A \\
\hline VIII & 130 & resolutaque crinem // euasit & S.D. \\
\hline IX & 260 & deflendaque facta $/ / \ldots$ pandunt & A \\
\hline XI & 2 & Sarranaque castra, // expediam: & B \\
\hline XI & 556 & fraternaque corda $/ /$... agnoscas & A \\
\hline XII & 5 & uicinaque late $/ / \ldots$ quatit & S.D. \\
\hline${ }^{*} \mathrm{XII}$ & 245 & uiolataque ceruix $/ /$... recumbit & S.D. \\
\hline XIV & 351 & constructaque saxa $/ / \ldots$ traxisse & S.D. \\
\hline XIV & 582 & inimicaque pestis, $/ / \ldots$ rapuisset $(584)$ & S.D. \\
\hline XIV & 632 & inhonoraque busta // respiciunt, & B \\
\hline XVI & 252 & extentaque tellus // Syrtibus & B \\
\hline XVI & 687 & consultaque uestra $/ / \ldots$ fecere & S.D. \\
\hline XVII & 103 & semustaque castra $/ / \ldots$ uolitant & S.D \\
\hline XVII & 476 & ipsaque ob ora $/ / \ldots$ micat & A \\
\hline
\end{tabular}

También en estos casos podemos establecer la distinción entre encabalgamientos en braquistiquio, abruptos desinentes y suaves desinentes, que habiamos contemplado en el tipo anterior formulado mediante sirremas nominales; asimismo aparecen señalados en nuestro texto con las letras «B», «A» $\mathrm{y}$ «S.D.».

En cuanto a la configuración del braquistiquio en este grupo, cabe señalar su reiterada vinculación con las formas verbales, que lo formulan en cinco ocasiones de las seis registradas por nosotros.

Conviene además reconocer una estructura que se repite en dos ocasiones, vv. I 70 y XI 2, donde se establece una relación vertical entre la forma verbal en braquistiquio y el término inicial de un verso anterior.

En I 70 el final Saturniaque arua representa un complemento de dirección hacia el que se vierte la acción de addiderat; con todo, el punto de mayor expresividad emerge de la triple relación hanc rabiem-in $\mathrm{Sa}$ turniaque arua-addiderat, donde el complemento de objeto, en comienzo de verso, se relaciona con el verbo, para mayor ornamentación, en una correspondencia vertical.

Con respecto al verso XI 2, el sintagma Sarranaque castra, otro acusativo de dirección situado en la cláusula, presta final a una secuencia de casi dos versos que encierra el complemento objeto de un verbo, expediam, destacado en braquistiquio a comienzos del v. 3; dicha forma 
verbal aparece introducida por la secuencia nunc age, que, a su vez, ocupa el inicio del v. 1; con ello se establece de nuevo un esquema de relación vertical.

En algún caso el autor procura la relación vertical también entre los dos términos del sirrema encabalgado; así en los versos V 628 y XII 245 , que aparecen señalados por un asterisco. Concretamente, en el ejemplo recogido en el libro $\mathrm{V}$, esta triple caraterización - cláusula anómala, encabalgamiento y relación vertical - marca otros tantos hitos con los que reforzar el fuerte efecto expresivo que consigue el autor por la paréquesis del sonido $\underline{\mathrm{t}}$, subrayando así el fragor del combate que comienza:

iactațus tițubante solo tremebundaque tela subducțaque tellure ruens, torquebaț in hostem,

Carácter un poco diverso presenta aquella cláusula cuyo encabalgamiento se confirma por la figura del bikolon:

XIV 272 Cossyraque parua // nec maior

donde las especificaciones parua y nec maior Megara se apoyan y se hacen más significativas por su interrelación.

La última disposición que se contempla en el encabalgamiento simple combinado con la cláusula tipo armaque nostra, se realiza por la disyunción entre una oración subordinada y la principal correspondiente; es lo que encontramos en los pasajes siguientes:

$\begin{array}{lll}\text { III } & 177 & \text { memorandaque uirtus, // quod ... } \\ \text { X } & 131 & \text { iraque anhelat, // } \mathrm{ni} . . . \\ \text { XVI } & 109 & \text { animosaque uirtus, // ni }\end{array}$

Con respecto a III 177 hay que recalcar que el verso 178 representa justamente el desarrollo del pronombre id que encabeza la frase anterior, razón por la cual ambas líneas aparecen estrechamente vinculadas.

En los versos X 131 y XVI 109 la expresión queda en suspenso, pues la prótasis del período hipotético «necesita" el enunciado de la apódosis correspondiente. En este caso debemos hablar, al menos, de encabalgamiento laxo.

El encabalgamiento doble es fórmula vinculada también al tipo armaque nostra en Silio Itálico, y se configura sobre la estructura del quiasmo, ya sea de carácter entrecruzado o concéntrico, como sucedía en relación con la cláusula tipo si bona norint. 
Los versos en que aparece son los siguientes:

$\begin{array}{ll}\text { *I } & 283 \\ { }^{*} \text { II } & 193 \\ \text { II } & 473 \\ \text { III } & 394 \\ \text { V } & 83 \\ \text { V } & 438 \\ \text { VI } & 50 \\ \text { VIII } & 365 \\ \text { IX } & 406 \\ \text { X } & 456 \\ \text { *XIV } & 214 \\ \text { *XV } & 26\end{array}$

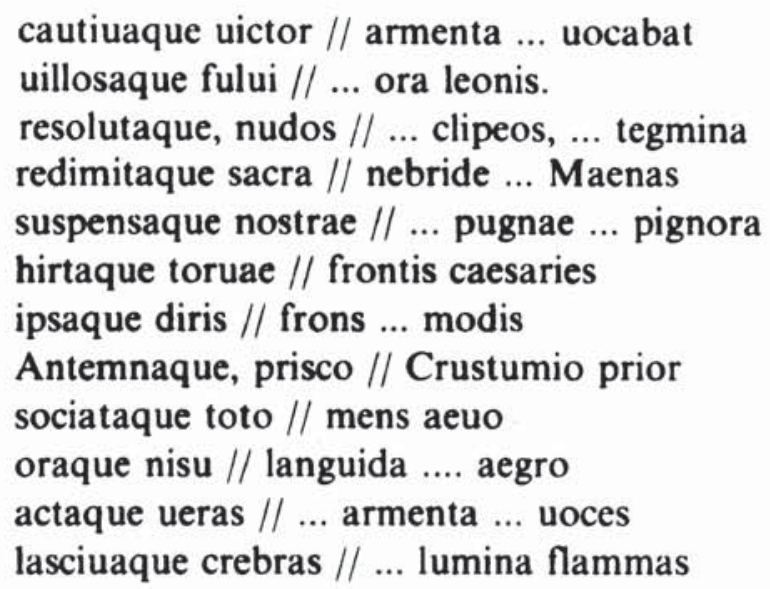

En los doce versos en los que Silio Itálico propone un encabalgamiento doble junto a la fórmula armaque nostra, se inclina casi definitivamente hacia la figura del encabalgamiento geminado, con una única excepción, el verso I 283. En él el autor disgrega, por una parte, el sintagma formado por adjetivo y sustantivo (captiuaque armenta) y por otra, el predicativo y el verbo correspondiente (uictor uocabat), relacionando además estos términos en vertical como broche de los dos versos correlativos.

Por lo demás, los encabalgamientos geminados pueden presentar tanto disposición quiasmática de ordenación entrecruzada, como concéntrica.

Ordenación entrecruzada tenemos en los versos: II 193, VI 50, IX 406, X 456, XIV 214 y XV 26.

Presentan quiasmo de ordenación concéntrica las líneas: II 473, V 13, V 438 y VIII 365.

Silio Itálico suele situar las formas determinantes en el verso que encabalga y los sustantivos en el encabalgado, de acuerdo con las normas del orden de palabras latino. La única excepción aparece en X 456, donde oraque y nisu quedan en expectativa ante la frontera versal, seguramente para destacar la imagen de extrema debilidad de Clelio, condición que aparece de manifiesto también a lo largo de todo el verso 457: languida uix aegro et dubia ceruice leuabat.

Resulta interesante constatar cómo el poeta añade, en un 25 por 100 de los casos, a la figura del quiasmo y a la anomalía clausular, el ornamento de la relación vertical: los términos de los sirremas que se disgregan aparecen situados en las cláusulas de dos versos consecutivos, con un dispositivo estudiado por el poeta para conseguir una mayor relevancia estética del texto. 
Los versos que presentan esta triple caracterización están señalados en el texto que ofrecemos con un asterisco. Entre ellos destacan, por el triple juego de aliteración (.), paréquesis $(-)$ y homeoteleuto $(=)$ que presentan, los versos XV 26-27:

\section{fronte deçor quaesitus açu, lasçiuaque çrebras ançipiti moțu iaçiebant lumina flammas}

Tras el estudio de estas cláusulas que revelan la disposición armaque nostra, podemos afirmar que esta fórmula debía de ser especialmente grata al poeta, ya que es entre las anómalas la que alcanza una mayor proporción de uso $(0,74$ por 100$)$, relativamente próxima al 1,04 por $100 \mathrm{del}$ autor de la Eneida. El origen de esta preferencia - en nuestra opinión - podría haber descansado en la caracterización de este tipo como elemento especialmente representativo en la dicción épica ${ }^{15}$.

Silio Itálico, en la configuración de esta cláusula, parece que siguió de cerca al poeta modélico, pues, como él, la vincula reiteradamente al encabalgamiento, de manera que un 74,15 por 100 de los finales tipo armaque nostra presentan discoincidencia de pausa versal y de sentido.

Su diseño más común realiza la disyunción del sirrema adjetivo-sustantivo con 27 ejemplos, en los que conserva el orden habitual de los elementos salvo en tres ocasiones: en dos de ellas el determinante es una forma participial (II 331 y XIII 72), y en la tercera está formado por un sintagma en genitivo (III 666).

El otro tipo que aparece casi con análoga frecuencia (23 ejemplos), establece la separación entre el verbo y su sujeto o alguno de sus elementos. La forma verbal se ofrece a veces en braquistiquio en el verso encabalgado, con lo que resulta doblemente expresivo, pero no es fórmula obligatoria; generalmente se conserva el orden habitual en latín, salvo en el verso I 618, en que la forma verbal ocupa el verso encabalgante y el ablativo agente el encabalgado: la excepción no lo es tanto, en el sentido de que en realidad nos encontramos ante una fórmula participial, a caballo entre el nombre y el verbo.

De modo semejante a lo que ocurre con la cláusula si bona norint, Silio Itálico innova provocando encabalgamientos dobles con la figura del quiasmo. El encabalgamiento en este caso es del tipo de los gemina-

15 Ya de por sí la presencia de la partícula -que parece hacer una especial referenica al lenguaje de la épica, como sugiere J. Hellegouarc'h en Le monosyllabe dans l'hexamètre latin, París 1964 , p. 259 ss. Esta misma sugerencia puede aplicarse a las cláusulas tipo armaque nostra, según se desprende de nuestro trabajo anteriormente citado. 
dos (única excepción en I 283) y se realiza indistintamente con ordenación entrecruzada o concéntrica. Conservando la norma de la lengua latina los adjetivos aparecen en el verso encabalgante y los sustantivos en el encabalgado con la única excepción de la línea $X 456$, ya comentada por nosotros.

En un 25 por 100 de los casos el poeta realiza esta figura aplicando el esquema de la relación vertical entre los dos términos del sirrema, que suele adornarse, por otra parte, con fenómenos del tipo aliteración, paréquesis u homeoteleuto.

Así en:

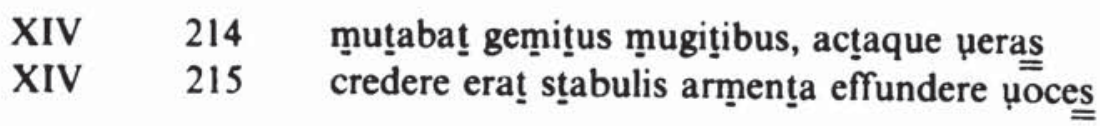

El hecho de que este tipo clausular aparezca unido repetidamente al encabalgamiento y esté rodeado de tantos cuidados desde el punto de vista estilístico nos hace suponer que, para el poeta, no se trataba de un final de verso de carácter banal; más bien parece que por alguna razón - quizá por su carácter representativo de la expresión épica - debía aparecer ante sus ojos como especialmente significativo. Con seguridad nos encontramos aquí ante otra manifestación de la imitatio uergiliana, ya que el autor de la Eneida realiza, de forma casi sistemática, la vinculación entre este tipo de cláusula y el encabalgamiento, aprovechando seguramente el desconcierto ${ }^{16}$ que produciría en el oyente o lector la ruptura de la secuencia acentual esperada en final de verso.

La novedad que el autor de los Punica introduce se concreta en su preferencia por los encabalgamientos geminados y por esa serie de fenómenos con que los rodea, en aras de una mayor expresividad.

\section{Conclusiones}

1. Del total de los 12.202 hexámetros que componen los Punica, la gran mayoría construye la cláusula con las tipologías habituales o regularizadas: $2+3,3+2$ ó $2+1+2$. En esto Silio Itálico se aleja un tanto de la práctica virgiliana, pues presenta en su obra un número de variaciones muy restringido: 192 , que suponen un 1,57 por 100 sobre el total

${ }^{16}$ Acerca del binomio desconcierto-esclarecimiento en la expresión poética y su relación con el encabalgamiento, cf. J. A. Martínez, Propiedades del lenguaje poético, Oviedo 1975. 
de sus versos, cifra notablemente inferior al 3,69 por 100 que aporta la obra de Virgilio.

2. Silio Itálico utiliza, para introducir variedad en la cláusula del hexámetro, casi todos los tipos «anómalos» presentes en el poeta modélico, a excepción del denominado medium secat agmen.

Las distribuciones «irregulares» en Silio y su repartición se establecen del modo siguiente:

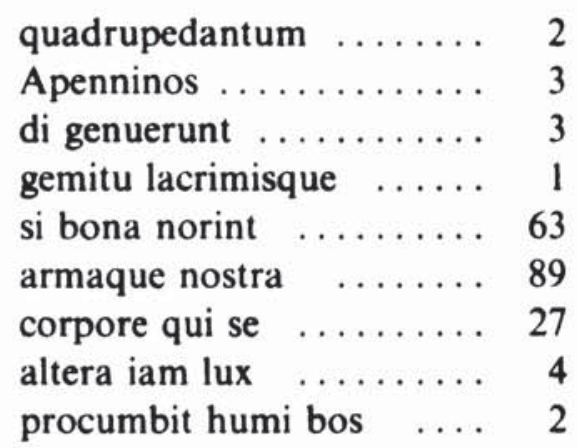

3. Los tipos con monosílabo final en relevancia y los que presentan un vocablo de gran volumen fónico - tetrasílabos o pentasílabosaparecen con carácter excepcional. En ellos podemos percibir una doble influencia: por una parte la virgiliana, ya que Silio Itálico recoge en gran medida los términos utilizados por el autor de la Eneida y los aplica con sus mismas técnicas, y por otra parte la de Ovidio, ya que elige algunos de los términos consagrados en este lugar del hexámetro por el autor de las Metamorfosis y rebaja - como él-, notablemente, el número de cláusulas con estas características.

4. Los únicos tipos "anómalos» productivos son los representados por los lemas si bona norint y armaque nostra, si bien sus proporciones se muestran inferiores a las que presenta el poeta modélico. En ambos casos, sin embargo, aparece patente la impronta virgiliana, pues Silio Itálico los construye según las directrices de Virgilio: las cláusulas $s i$ bona norint y armaque nostra aparecen vinculadas de modo casi sistemático al encabalgamiento, dando, además, lugar la primera a una reiterada diéresis bucólica.

5. Silio Itálico polariza el uso de estas cláusulas anómalas más repetidas -en relación al tipo de encabalgamiento con el que se vinculan- de la siguiente manera:

a) el tipo si bona norint se aprovecha sobre todo para introducir encabalgamientos suaves y desinentes;

b) el tipo armaque nostra, en cambio, parece más adecuado - según su utilización en los Punica - para proponer encabalgamientos desinentes abruptos, con eficaz uso del braquistiquio, que subraya el tema patético o sobrenatural. 
6. Con todo, la principal innovación que aporta Silio Itálico se refiere a la formulación de los tipos si bona norint y armaque nostra con el concurso de encabalgamientos complejos (en el tipo con enclítica casi siempre geminados), realzados con la figura del quiasmo, bien sea de ordenación entrecruzada o concéntrica.

Un último motivo de ornamentación - propio de la técnica de $\mathrm{Si}$ lio - se refiere a la relación vertical que establece, al situar en las cláusulas de dos versos consecutivos los miembros de los sirremas en disyunción.

En ambos tipos clausulares, pero mucho más marcadamente con la fórmula tipo armaque nostra, esta conjunción de fenómenos estilísticos - el uso de una cláusula anómala y su proyección sobre un encabalgamiento complejo en figura de quiasmo - suele además ilustrarse con la presencia de otros índices de carácter fonético-estilistico, como la paréquesis, la aliteración o el homeoteleuto.

Silio Itálico, al adoptar este tipo de finales, ha sabido encontrar una cláusula sumamente elaborada, casi barroca, en perfecta conjunción con los gustos de la época.

M. a Luisa Arribas Hernáez 